\title{
Effects of Training with a Brain-Computer Interface- Controlled Robot on Rehabilitation Outcome in Patients with Subacute Stroke: A Randomized Controlled Trial
}

\author{
Chen-Guang Zhao · Fen Ju • Wei Sun · Shan Jiang • Xiao Xi • \\ Hong Wang · Xiao-Long Sun · Min Li · Jun Xie · Kai Zhang • \\ Guang-Hua Xu $\cdot$ Si-Cong Zhang $\cdot$ Xiang Mou $\cdot$ Hua Yuan (D)
}

Received: January 1, 2022 / Accepted: January 25, 2022 / Published online: February 16, 2022

(C) The Author(s) 2022

\section{ABSTRACT}

Introduction: Stroke is always associated with a difficult functional recovery process. A brain-computer interface (BCI) is a technology which provides a direct connection between the human brain and external devices. The primary aim of this study was to determine whether training with a BCI-controlled robot can improve functions in patients with subacute stroke.

Methods: Subacute stroke patients aged 32-68 years with a course of 2 weeks to 3 months were randomly assigned to the BCI group or to the sham group for a 4 -week course. The primary outcome measures were Loewenstein Occupational Therapy Cognitive Assessment (LOCTA) and Fugl-Meyer Assessment for Lower

Chen-Guang Zhao and Fen Ju contributed equally to this article as the co-first authors.

C.-G. Zhao · F. Ju · W. Sun · X. Xi · H. Wang ·

X.-L. Sun $\cdot$ X. Mou $\cdot$ H. Yuan $(\bowtie)$

Department of Rehabilitation Medicine, Xijing

Hospital, Fourth Military Medical University, Xi'an,

China

e-mail: yuanhua_fmmu@hotmail.com

S. Jiang

Department of Rehabilitation Medicine, China-

Japan Friendship Hospital, Beijing, China

M. Li · J. Xie · K. Zhang · G.-H. Xu - S.-C. Zhang School of Mechanical Engineering, Xi'an Jiaotong University, Xi'an, China
Extremity (FMA-LE). Secondary outcome measures included Fugl-Meyer Assessment for Balance (FMA-B), Functional Ambulation Category (FAC), Modified Barthel Index (MBI), serum brain-derived neurotrophic factor (BDNF) levels and motor-evoked potential (MEP).

Results: A total of 28 patients completed the study. Both groups showed a significant increase in mean LOCTA (sham: $P<0.001$, Cohen's $d=-2.972$; BCI: $P<0.001$, Cohen's $d=-4.266$ ) and FMA-LE (sham: $P<0.001$, Cohen's $d=-3.178$; BCI: $P<0.001$, Cohen's $d=-3.063)$ scores. The LOCTA scores in the BCI group were $14.89 \%$ higher than in the sham group $(P=0.049$, Cohen's $d=-0.580)$. There were no significant differences between the two groups in terms of FMA-B $(P=0.363$, Cohen's $d=-0.252), \quad$ FAC $\quad(P=0.363), \quad$ or $\quad$ MBI $(P=0.493$, Cohen's $d=-0.188)$ scores. The serum levels of BDNF were significantly higher within the BCI group $(P<0.001$, Cohen's $d=-1.167)$, and the MEP latency decreased by $3.75 \%$ and $4.71 \%$ in the sham and BCI groups, respectively.

Conclusion: Training with a BCI-controlled robot combined with traditional physiotherapy promotes cognitive function recovery, and enhances motor functions of the lower extremity in patients with subacute stroke. These patients also showed increased secretion of BDNF.

Trial Registration: Chinese clinical trial registry: ChiCTR-INR-17012874. 
Keywords: Brain-computer interface; Robot training; Stroke; Rehabilitation; Randomized controlled trial

\section{Key Summary Points}

The aim of the study was to evaluate the effects of training with a brain-computer interface (BCI)-controlled robot on rehabilitation outcome in patients with subacute stroke.

This randomized clinical trial with 28 patients with subacute stroke showed that, to some extent, training with a BCIcontrolled robot (combined with traditional physiotherapy) effectively promotes cognitive recovery, and enhances motor function of the lower extremities. These results were accompanied by increased secretion of brain-derived neurotrophic factor (BDNF).

This means that training with a BCIcontrolled robot may be a safe and effective strategy in subacute stroke rehabilitation.

\section{INTRODUCTION}

Stroke is one of the most common causes of disability and death, with high mortality and disability rates [1]. The 2016 Global Burden of disease data showed that stroke is the main causes of annual loss of life in our country [2,3]. Recovery of neural function after stroke is a difficult process [4]. Traditional stroke rehabilitation programs include a variety of treatment strategies, with varying degrees of evidencebased support $[5,6]$. Usually, these treatments include repetitive physical exercises aimed at restoring muscle strength and the activities of daily living [7]. In the early phase, the treatment may include passive exercises to maintain the integrity of physical structures, in preparation for neurological recovery [8]. As the rehabilitation course continues, exercises gradually shift to active-assistive training. The limitations of passive training during the early stage of stroke rehabilitation prompted us to investigate innovative approaches that would enable patients to exercise at this stage of their disease [9].

A brain-computer interface (BCI) is a technology that bypasses normal nervous and muscle pathways, providing a direct connection between the human brain and external devices $[10,11]$. BCI training systems can use EEG signals from motor imagery performance with sensory real-time feedback and decode these signals to enable patients to direct devices, such as wheelchairs, robots, and prosthetic devices, including exoskeletons. In a previous study, we, for the first time, proposed a special form of visual stimulation, involving non-directional specific motion reversal, to elicit steady-state motion visual-evoked potentials (SSMVEPs) [12-14]. This has BCI applicability in patients with different neurophysiological impediments, and is associated with stronger responses and less visual discomfort [15]. Based on this technology [16], we developed a BCI-controlled robot for lower limb rehabilitation of patients with subacute stroke.

We aimed to compare the recovery of subacute stroke patients treated with the BCI-controlled robot coupled with physiotherapy (PT), with traditional training via a randomized trial. We hypothesized that training with the BCIcontrolled robot would improve lower limb motor function, and trigger endocrine and neurophysiological changes in patients with subacute stroke.

\section{METHODS}

\section{Standard Protocol Approvals and Registrations}

This study was a single-center, randomized, double-blind, sham-controlled clinical trial with two parallel arms. The study was approved by the Institute Ethics Committee of Xijing Hospital (KY20172051), adhered to the guidelines of the Declaration of Helsinki, and was registered in the Chinese Clinical Trial Registry 
(ChiCTR-INR-17012874). Informed consent was received to participate, and also included the statement we provided concerning written informed consent for the publication and for the photograph (Fig. 1A) included in the paper.

\section{Participants}

Among 33 patients assessed for eligibility, a total of 31 [mean $( \pm \mathrm{SD})$ age, $52.4( \pm 11.2)$ years, including 27 men (87.1\%)], were recruited for the study between October 2017 and December 2018 at the Department of Rehabilitation of Xijing Hospital (Table 1).

The inclusion criteria were: (1) first onset, based on the diagnostic criteria for stroke; (2) computed tomography (CT) or magnetic resonance imaging (MRI) localization of the lesion unilaterally in the basal ganglia, internal capsule, cortex, or subcortical area. The course of the disease was in subacute phase which was from 2 weeks to 3 months with stable vital signs; (3) between 30 and 70 years of age; (4) ability to comprehend instructions (Mini-Mental State Examination score $\geq 22$ ); and (5) signed informed consent form.

The exclusion criteria were as follows: (1) unstable clinical progression secondary to brain damage; (2) history of epilepsy; (3) severe cardiopulmonary insufficiency resulting in the patient not being able to tolerate the treatment; (4) unilateral neglect; (5) history of eye disease affecting visual acuity; and (6) motor dysfunction due to other causes.

The commercial software G Power was used to achieve a statistical power of 0.8 , with

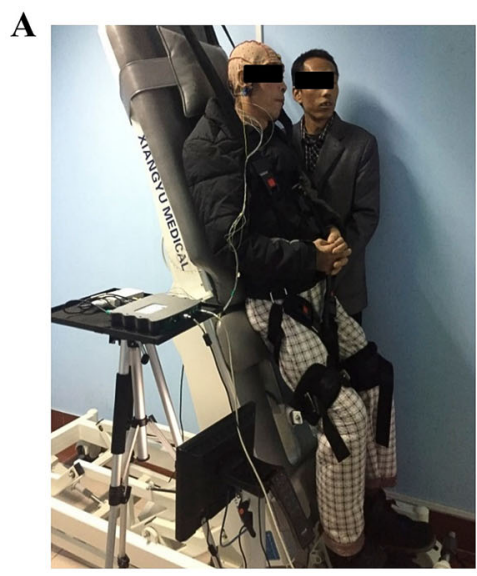

B

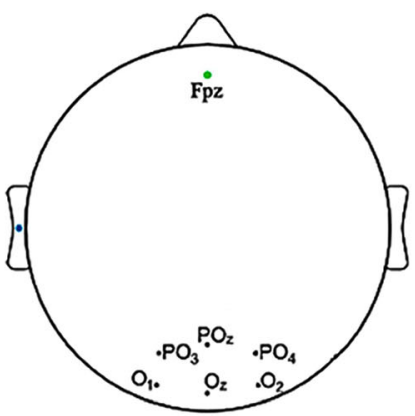

C

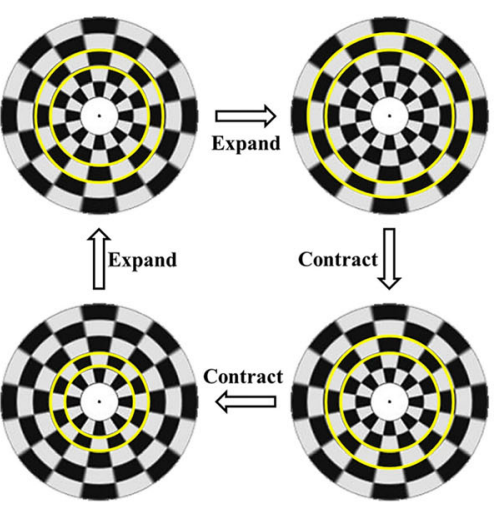

D

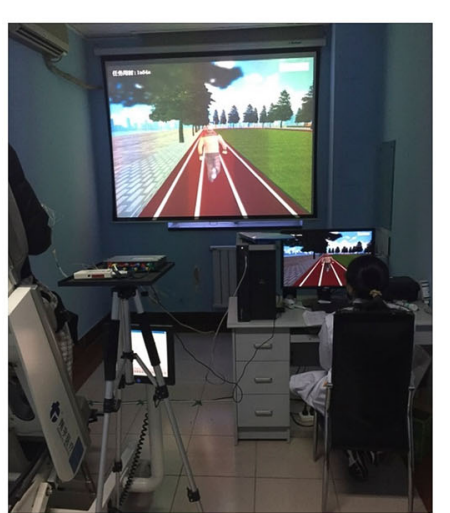

$\mathbf{E}$

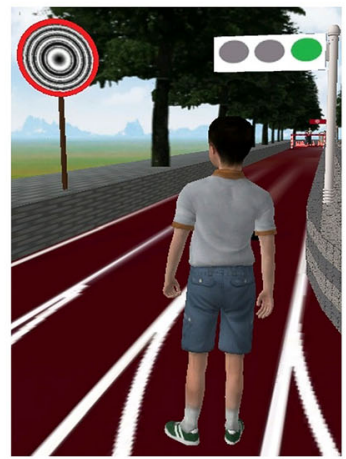

Level 1

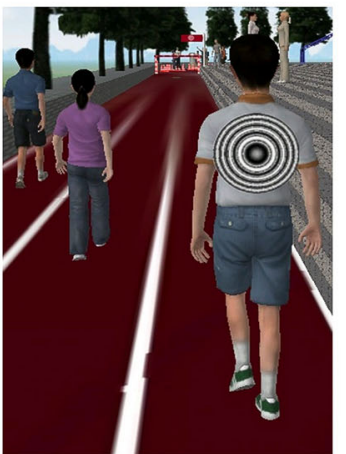

Level 2

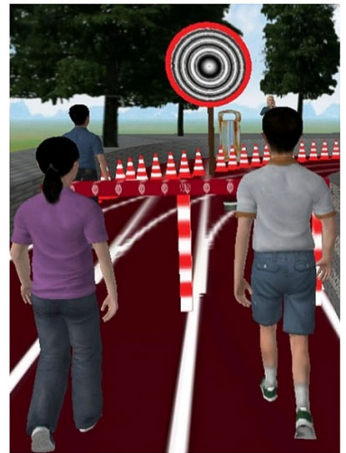

Level 3
Fig. 1 Training procedure. Participants were placed on the training robot $(\mathbf{A})$ and EEGs were recorded using the international 10-20 system (B). A Newton's ring was used as a stimulator $(\mathbf{C})$, enabling the participants to control the robot (D) and to exercise with varying levels of difficulty (E) 
Table 1 Baseline participant characteristics

\begin{tabular}{|c|c|c|c|c|}
\hline Characteristics & $\begin{array}{l}\text { Total } \\
(n=28)\end{array}$ & $\begin{array}{l}\text { Sham group } \\
(n=14)\end{array}$ & $\begin{array}{l}\text { BCI group } \\
(n=14)\end{array}$ & $P$ value \\
\hline Age, years; mean $\pm S D$ & $53.1 \pm 11.5$ & $56.1 \pm 11.5$ & $50.1 \pm 11.1$ & 0.178 \\
\hline Sex, $n(\%)$ & & & & 1 \\
\hline Male & $25(89.3 \%)$ & $12(85.7 \%)$ & $13(92.9 \%)$ & \\
\hline Female & $3(10.7 \%)$ & $2(14.3 \%)$ & $1(7.1 \%)$ & \\
\hline Stroke type, $n(\%)$ & & & & 0.270 \\
\hline Ischemic & $14(50.0 \%)$ & $9(64.3 \%)$ & $5(35.7 \%)$ & \\
\hline Hemorrhagic & $14(50.0 \%)$ & $5(35.7 \%)$ & $9(64.3 \%)$ & \\
\hline Affected hemisphere, $n$ (\%) & & & & 0.266 \\
\hline Left & $14(50.0 \%)$ & $5(35.7 \%)$ & $9(64.3 \%)$ & \\
\hline Right & $14(50.0 \%)$ & $9(64.3 \%)$ & $5(35.7 \%)$ & \\
\hline Time since stroke, days; mean $\pm S D$ & $37.3 \pm 40.2$ & $27.5 \pm 18$ & $47.1 \pm 53.2$ & 0.202 \\
\hline LOCTA, mean \pm SD & $56.4 \pm 17.2$ & $57.1 \pm 17.3$ & $55.8 \pm 17.8$ & 0.848 \\
\hline FMA-LE, mean $\pm S D$ & $10.3 \pm 6.2$ & $10.1 \pm 6.8$ & $10.4 \pm 5.7$ & 0.882 \\
\hline FMA-B, mean $\pm S D$ & $4.0 \pm 2.7$ & $4.1 \pm 2.8$ & $3.9 \pm 2.7$ & 0.839 \\
\hline FAC & & & & 1 \\
\hline Level 0 & $18(64.3 \%)$ & $9(64.3 \%)$ & $9(64.3 \%)$ & \\
\hline Level 1 & $4(14.3 \%)$ & $2(14.3 \%)$ & $2(14.3 \%)$ & \\
\hline Level 2 & $5(17.9 \%)$ & $2(14.3 \%)$ & $3(21.4 \%)$ & \\
\hline Level 3 & $1(3.6 \%)$ & $1(3.6 \%)$ & $0(0.0 \%)$ & \\
\hline Level 4 & $0(0.0 \%)$ & $0(0.0 \%)$ & $0(0.0 \%)$ & \\
\hline Level 5 & $0(0.0 \%)$ & $0(0.0 \%)$ & $0(0.0 \%)$ & \\
\hline $\mathrm{MBI}$, mean $\pm \mathrm{SD}$ & $24.4 \pm 13.9$ & $24.9 \pm 14.0$ & $23.8 \pm 14.3$ & 0.832 \\
\hline $\mathrm{BDNF}$, mean $\pm \mathrm{SD}$ & $89.5 \pm 19.0$ & $88.9 \pm 19.9$ & $90.1 \pm 18.8$ & 0.868 \\
\hline Latency, mean $\pm S D$ & $25.6 \pm 3.1$ & $25.7 \pm 3.22$ & $25.5 \pm 3.25$ & 0.902 \\
\hline Amplitude, mean \pm SD & $344 \pm 262$ & $333 \pm 257$ & $356 \pm 285$ & 0.963 \\
\hline
\end{tabular}

statistical significance at $P<0.05$ (two-tailed test) and an effect size of 0.45 , a minimal sample size of 28 patients, which were required for the present trial.

\section{Randomization and Blinding}

A computer random number generator assigned patients to the BCI-controlled robot group (BCI group for short) or to the sham group, using a block randomization strategy. Before the recruitment phase, sealed envelopes containing 
the protocol materials and patient allocation were prepared and numbered sequentially. After patients agreed to participate in the trial, the envelopes were opened sequentially by the coordinator, who was not involved with the patient's intervention. Patients were evaluated at different time points: before intervention (T0), and 1 week (T1), 2 weeks (T2), and 4 weeks (T3) afterwards. Biochemical and neurophysiological measurements were performed only at TO and T3. All investigators were rehabilitation science majors with more than 3 years of professional experience. To guarantee that the study was performed in a double-blind manner, two independent, experienced therapists evaluated the outcome. Participants were unaware of the group to which they had been allocated.

\section{Intervention}

Participants were asked to lie comfortably on the training robot with the help of therapists in a quiet, ordinary well-illuminated room with no electromagnetic shielding. The training robot then moved from the stopping (horizontal) to the working (vertical) position (Fig. 1A). We adopted the international 10-20 system for EEG recordings, which included $\mathrm{O} 1, \mathrm{O} 2, \mathrm{Oz}, \mathrm{PO} 3$, PO4, and POz sites (Fig. 1B). The EEG signals were referenced to a unilateral earlobe, grounded at the frontal position (Fpz), and sampled at $1200 \mathrm{~Hz}$ using a g.USBamp (g.tec, Austria) system. Signals were online band-pass filtered from 1 to $100 \mathrm{~Hz}$ and notch filtered between 48 and $52 \mathrm{~Hz}$ to remove artifacts and power line interference. All electrode impedances were kept below $5 \mathrm{kV}$ during the experiments.

After placing the electrodes, the participants were asked to view the screen binocularly and to fixate on the center of the target stimulator until the training started. We used Newton's ring as a stimulator, as described before [15]. Briefly, the phase of the Newton's ring was temporally sinusoidally shifted to generate the motion reversal procedure, which included alternating inward contraction and outward expansion motions (Fig. 1C). After gazing at the Newton's ring for $1 \mathrm{~s}$ (which shifted at a specific frequency), the SSMVEPs of the patients were recorded. Each specific frequency signal was assigned a movement task (e.g., starting to walk, accelerated walking, stopping), and the training robot moved correspondingly with the movement task (Fig. 1D). We set different levels of training tasks for each patient, so that they could challenge themselves, step by step, with different tasks, and to improve compliance and patient interest (Fig. 1E). For example, in level 1, the patients could start a training game with the BCI system. In level 2, they manipulated running with left/right turn or accelerating, while, in level 3, they could compete running with obstacles. In the sham group, the EEG recording system was placed, but it was not operational. The patients also exercised on the training robot while observing the avatar moving on a screen which was not controlled by BCI system. Besides training with the BCI-controlled robot, conventional PT and medical treatments (e.g., management of high blood pressure) were also provided to the participants. Patients in the BCI and sham groups received $30 \mathrm{~min}$ of robot training and $30 \mathrm{~min}$ of PT training once a day, 6 days a week, for a total of 4 weeks.

\section{Motor-Evoked Potentials (MEPs) Recording}

MEPs (latency and maximal amplitude) were recorded, as described in our previous study [17]. Briefly, single pulse TMS (YIRUIDE Medical Equipment, China) was applied over the affected hemisphere at $70-85 \%$ intensity of the machine export threshold. The MEP latency and maximal amplitude were calculated based on five waves showing large amplitude and good repeatability. MEP latency is defined as the interval between the start of the single pulse TMS on M1 and the initiation of the EMG response (ms) in the contralateral target muscle. MEP amplitude is defined as the peak-to-peak amplitude $(\mathrm{mV})$ in the EMG response of the contralateral FDI. A TMS-elicited MEP was categorized as present or absent $[18,19]$. 


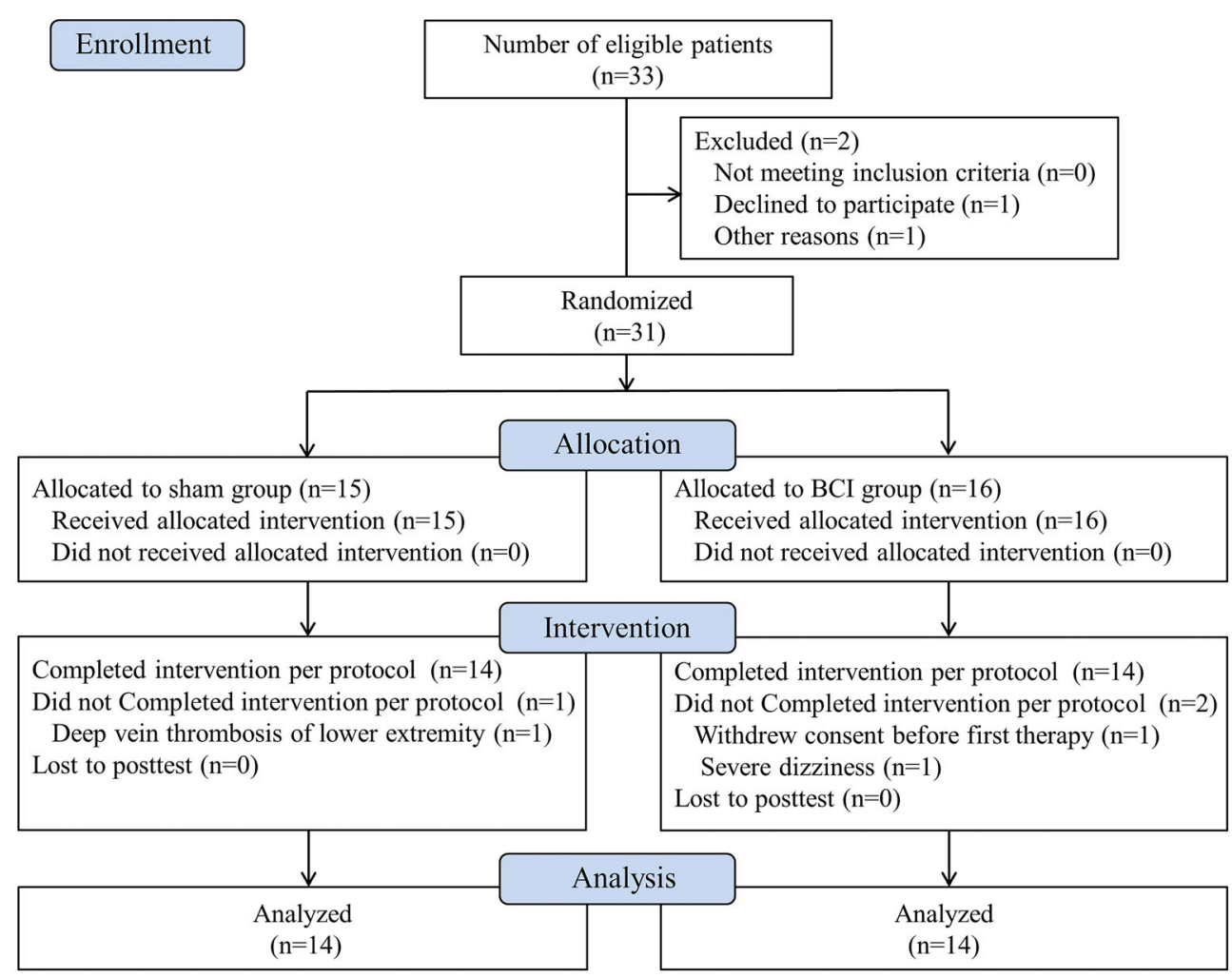

Fig. 2 CONSORT flow diagram for randomization of patients with subacute stroke

\section{Blood Collection and Measurement of BDNF Serum Levels}

Blood samples were collected in EDTA-treated tubes (BD, Franklin Lakes, NJ, USA) and immediately frozen at $-20^{\circ} \mathrm{C}$. Within 3 days of collection, samples were transferred to an ultra-low temperature freezer. Serum BDNF concentrations were measured by means of enzymelinked immunosorbent assay kits (Multi Sciences, Catalog Nos. EK11272, lower detection limit $=1.41 \mathrm{pg} / \mathrm{mL}$ ), according to the manufacturer's instructions.

\section{Clinical Assessment}

The primary outcomes were as follows:

(1) Loewenstein Occupational Therapy Cognitive Assessment (LOTCA): LOTCA is an accurate test for post-stroke patients with cognitive impairment [20], measuring areas such as orientation ability, visual perception, spatial perception, praxis, visuomotor organization, thinking operations, memory, attention, and absorption $[21,22]$. A higher value indicates better cognition.

(2) Fugl-Meyer Assessment for Lower Extremity (FMA-LE): FMA is a performance-based, stroke-specific test to evaluate motor function (upper and lower extremity), sensory function, balance, joint motion range, and joint pain [23]. In this study, we focused mainly on FMA-LE and FMA-B (maximum scores: 34 and 14, respectively).

The secondary outcomes were as follows:

(1) Functional Ambulation Category (FAC): FAC was utilized to quickly assess the ambulation ability of the patients. Categories range from 0 (patient cannot walk or needs help from 2 or more persons) to 5 (patient can walk independently on uneven surfaces, and safely climb stairs and inclines) [24]. 
Table 2 Treatment outcomes between groups

\begin{tabular}{|c|c|c|c|c|c|c|c|}
\hline Treatment & $\begin{array}{l}\text { Mean }(\mathrm{SD}) \\
\text { before treatment } \\
\left(\mathrm{T}_{0}\right)\end{array}$ & $\begin{array}{l}\text { Mean }(S D) \text { after } \\
\text { treatment }\left(T_{3}\right)\end{array}$ & $\begin{array}{l}\text { Cohen's } \\
d\left(T_{0}-\mathrm{T}_{3}\right)\end{array}$ & $P$ value & $\begin{array}{l}\text { Mean } \\
\text { difference } \\
(95 \% \mathrm{CI})\end{array}$ & $\begin{array}{l}\text { Cohen's } \\
d \text { (sham to BCI } \\
\text { at } \mathrm{T}_{3} \text { ) }\end{array}$ & $P$ value \\
\hline
\end{tabular}

Primary outcomes

LOCTA

\begin{tabular}{|c|c|c|c|c|c|c|c|}
\hline Sham & $57.07(17.28)$ & $71.93(17.25)$ & -4.266 & $<0.001$ & $\begin{array}{l}-14.86 \\
(-17.75 \text { to } \\
-11.97)\end{array}$ & & \\
\hline $\mathrm{BCI}$ & $55.79(17.81)$ & $82.64(17.84)$ & -2.972 & $<0.001$ & $\begin{array}{l}-26.86 \\
(-34.36 \text { to } \\
-19.35)\end{array}$ & -0.580 & 0.049 \\
\hline
\end{tabular}

FMA-LE

$\begin{array}{cccccccc}\text { Sham } & 10.07(6.83) & 16.36(7.63) & -3.178 & <0.001 & -6.29(-7.75 \\ & & & \text { to }-4.82) & \\ \text { BCI } & 10.43(5.67) & 18.21(7.59) & -3.063 & <0.001 & -7.79(-9.67 & -0.168 & 0.540 \\ & & & & \text { to }-5.90)\end{array}$

Secondary outcomes

FMA-B

$\begin{array}{cccccccc}\text { Sham } & 4.07(2.84) & 8.50(3.01) & -3.365 & <0.001 & -4.43(-5.41 \\ & & & \text { to }-3.45) & \\ \text { BCI } & 3.86(2.68) & 9.57(3.03) & -3.396 & <0.001 & -5.71(-6.96 & -0.252 & 0.363 \\ & & & & \text { to }-4.46)\end{array}$

FAC

(median

(IQR))

$\begin{array}{llllllll}\text { Sham } & 0.00(1.00) & 1.00(2.00) & \text { NA } & <0.001 & \text { NA } & & \\ \text { BCI } & 0.00(1.00) & 2.00(2.00) & \text { NA } & 0.001 & \text { NA } & \text { NA } & 0.363\end{array}$

MBI

\begin{tabular}{|c|c|c|c|c|c|c|c|}
\hline Sham & $24.93(13.97)$ & $54.79(18.30)$ & -1.778 & $<0.001$ & $\begin{array}{l}-29.86 \\
(-43.80 \text { to } \\
-15.91)\end{array}$ & & \\
\hline $\mathrm{BCI}$ & $23.79(14.29)$ & $60.29(13.69)$ & -2.563 & $<0.001$ & $\begin{array}{l}-36.50 \\
(-48.33 \text { to } \\
-24.67)\end{array}$ & -0.188 & 0.493 \\
\hline
\end{tabular}

Serum BDNF concentrations

$$
\begin{array}{cccc}
\text { Sham } \quad 88.90(19.88) \quad 92.42(21.00) & -0.242 & 0.381 & -3.51 \\
& & (-11.88 \text { to } \\
& & 4.86)
\end{array}
$$


Table 2 continued

\begin{tabular}{|c|c|c|c|c|c|c|c|}
\hline Treatment & $\begin{array}{l}\text { Mean }(\mathrm{SD}) \\
\text { before treatment } \\
\left(\mathrm{T}_{\mathbf{0}}\right)\end{array}$ & $\begin{array}{l}\text { Mean (SD) after } \\
\text { treatment }\left(\mathrm{T}_{3}\right)\end{array}$ & $\begin{array}{l}\text { Cohen's } \\
d\left(T_{0}-\mathrm{T}_{3}\right)\end{array}$ & $P$ value & $\begin{array}{l}\text { Mean } \\
\text { difference } \\
(95 \% \mathrm{CI}) \\
\end{array}$ & $\begin{array}{l}\text { Cohen's } \\
d \text { (sham to BCI } \\
\text { at } \mathrm{T}_{3} \text { ) }\end{array}$ & $P$ value \\
\hline $\mathrm{BCI}$ & $90.14(18.81)$ & $106.09(20.36)$ & -1.167 & $<0.001$ & $\begin{array}{l}-15.95 \\
\quad(-23.84 \text { to } \\
-8.06)\end{array}$ & -0.661 & 0.092 \\
\hline \multicolumn{8}{|l|}{$\begin{array}{l}\text { MEP } \\
\text { latency }\end{array}$} \\
\hline Sham & $25.72(3.22)$ & $24.76(3.09)$ & 2.707 & $<0.001$ & $\begin{array}{c}0.97 \text { (0.69 to } \\
1.24)\end{array}$ & & \\
\hline $\mathrm{BCI}$ & $25.52(3.24)$ & $24.31(3.22)$ & 1.160 & 0.013 & $\begin{array}{l}1.21(0.34 \text { to } \\
2.09)\end{array}$ & 0.141 & 0.776 \\
\hline \multicolumn{8}{|c|}{ Maximal MEP amplitude } \\
\hline Sham & $333.22(257.07)$ & $371.78(282.32)$ & -0.541 & 0.143 & $\begin{array}{l}-38.56 \\
(-93.32 \text { to } \\
16.21)\end{array}$ & & \\
\hline $\mathrm{BCI}$ & $356.25(285.08)$ & $429.75(243.08)$ & -0.704 & 0.087 & $\begin{array}{l}-73.50 \\
\quad(-160.82 \text { to } \\
13.82)\end{array}$ & -0.219 & 0.659 \\
\hline
\end{tabular}

$S D$ standard deviation, $C I$ confidence interval, LOTCA Loewenstein Occupational Therapy Cognitive Assessment, FMA$L E$ Fugl-Meyer Assessment for Lower Extremity, FAC Functional Ambulation Category, FMA-B Fugl-Meyer Assessment for Balance, $M B I$ Modified Barthel Index, $B D N F$ serum brain-derived neurotrophic factor, $M E P$ Motor-evoked potential, $I Q R$ interquartile range

(2) Fugl-Meyer Assessment for Balance (FMAB)

(3) Modified Barthel Index (MBI): MBI is a widely used and reliable index assessing the activities of daily life [25], and includes 10 activities, such as dressing, bathing, and toilet. The highest score is 100, which indicates full independence.

(4) Serum BDNF levels.

(5) Neurophysiological parameters, including MEP latency and amplitude.

\section{Statistical Analysis}

The statistical analyses were performed using SPSS software (v.20.0; IBM, Chicago, IL, USA). The effects of training with the BCI-controlled robot on LOCTA, FMA, and MBI scores, as well as on biochemical and neurophysiological parameters, were analyzed by means of repeated measures analysis of variance, with time as the within-subjects factor and treatment as the between-subjects factor. Bonferroni adjustment was performed for post hoc analysis of multiple comparisons. For FAC analysis, we performed Friedman tests for within-group comparisons and Mann-Whitney $U$ tests for between-group comparisons. If the Friedman test showed statistically significant differences, Wilcoxon matched-pairs tests were performed to compare baseline with post-treatment (T0 with $\mathrm{T} 1, \mathrm{~T} 2$, or T3) values. A $P$ value less than 0.05 was considered statistically significant. We calculated Cohen's $d$ and $\eta^{2}$ as measures of effect sizes. The interpretation of Cohen's $d$ and $\eta^{2}$ is described in our previous paper [17]. Effect sizes were also calculated for the mean differences (MD) of the 


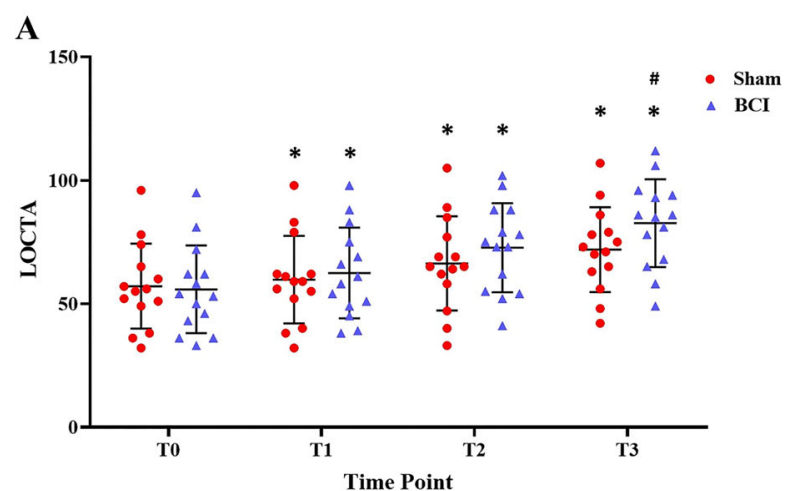

Fig. 3 Primary outcomes, including Loewenstein Occupational Therapy Cognitive Assessment (LOTCA) (A) and Fugl-Meyer Assessment for Lower Extremity (FMA-LE) (B) mean scores showed significant improvement of cognitive and motor function in both groups. However, only the BCI group showed a significant

assessment after training with the BCI-controlled robot or sham treatment.

\section{RESULTS}

The procedure was well tolerated, and no significant adverse effects were reported in either group. The two groups did not differ at baseline level (T0) with respect to age, gender, lesion side, diagnosis, or time since stroke. Three patients discontinued the treatment while 28 patients completed the treatments. One patient from the sham group had to abandon the study due to deep vein thrombosis of the lower extremity, while two other patients from the BCI group dropped out because of consent or dizziness. The remaining 28 patients [mean $( \pm \mathrm{SD})$ age: $53.1( \pm 11.5)$ years; 25 men $(89.3 \%)]$ completed the 4-week trial (Fig. 2).

\section{Primary Outcome}

\section{LOTCA}

The within-subject effects in the BCI group were significant $\left(P<0.001, \eta^{2}=0.854\right)$. The LOCTA score started to increase in the 1st week of the intervention [MD $-6.64(95 \%$ CI -9.24 to $-4.04)$, Cohen's $d=-2.124, P<0.001]$, and was highest in the 4 th week [MD, - 26.86 (95\%

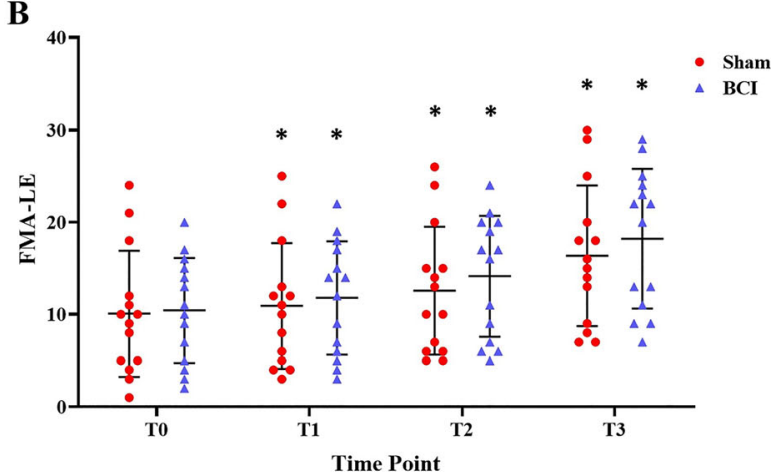

improvement in LOCTA scores in the 4th week of treatment. Error bars indicate the standard deviation (SD). Asterisks indicate significant pre-post differences (with respect to $T 0)\left({ }^{*} P<0.05\right)$. A pound sign $(£)$ indicates a significant difference when compared with the sham group $\left({ }^{\#} P<0.05\right)$

CI -34.36 to -19.35$)$, Cohen's $d=-2.972$, $P<0.001]$. Likewise, there were significant within-subject effects in the sham group $\left(P<0.001, \eta^{2}=0.890\right)$. The scores for the 1 st and 4th week were significantly higher than the baseline score $[\mathrm{MD}-2.64(95 \% \mathrm{CI}-5.18$ to $-0.11)$, Cohen's $d=-0.866, P=0.039]$ [MD - 14.86 (95\% CI - 17.75 to - 11.97), Cohen's $d=-4.266, \quad P<0.001] . \quad$ The interaction between time and intervention was significant $\left(P=0.003, \quad \eta^{2}=0.418\right)$. The LOCTA scores between the two groups were not significantly different until the 4 th week, when the scores in the BCI group were $14.89 \%$ higher than in the sham group [MD $-10.71(95 \% \mathrm{CI}-21.38$ to - 0.052), Cohen's $d=0.580, \quad P=0.049$ ] (Table 2; Fig. 3A).

\section{FMA-LE}

The interaction between time and intervention was not significant $\left(P=0.363, \eta^{2}=0.068\right)$. Compared with the baseline FMA-LE scores (before intervention), the scores in both the sham and BCI groups increased significantly as the treatment progressed, $\quad(P<0.001$, $\left.\eta^{2}=0.810 ; P<0.001, \eta^{2}=0.795\right)$. The highest FMA-LE scores were observed in the 4th week [MD - 6.29 (95\% CI - 7.75 to - 4.82), Cohen's $d=-3.178, P<0.001$; MD 7.79 (95\% CI -9.67 to -5.90$)$, Cohen's $d=-3.063, \quad P<0.001]$. 


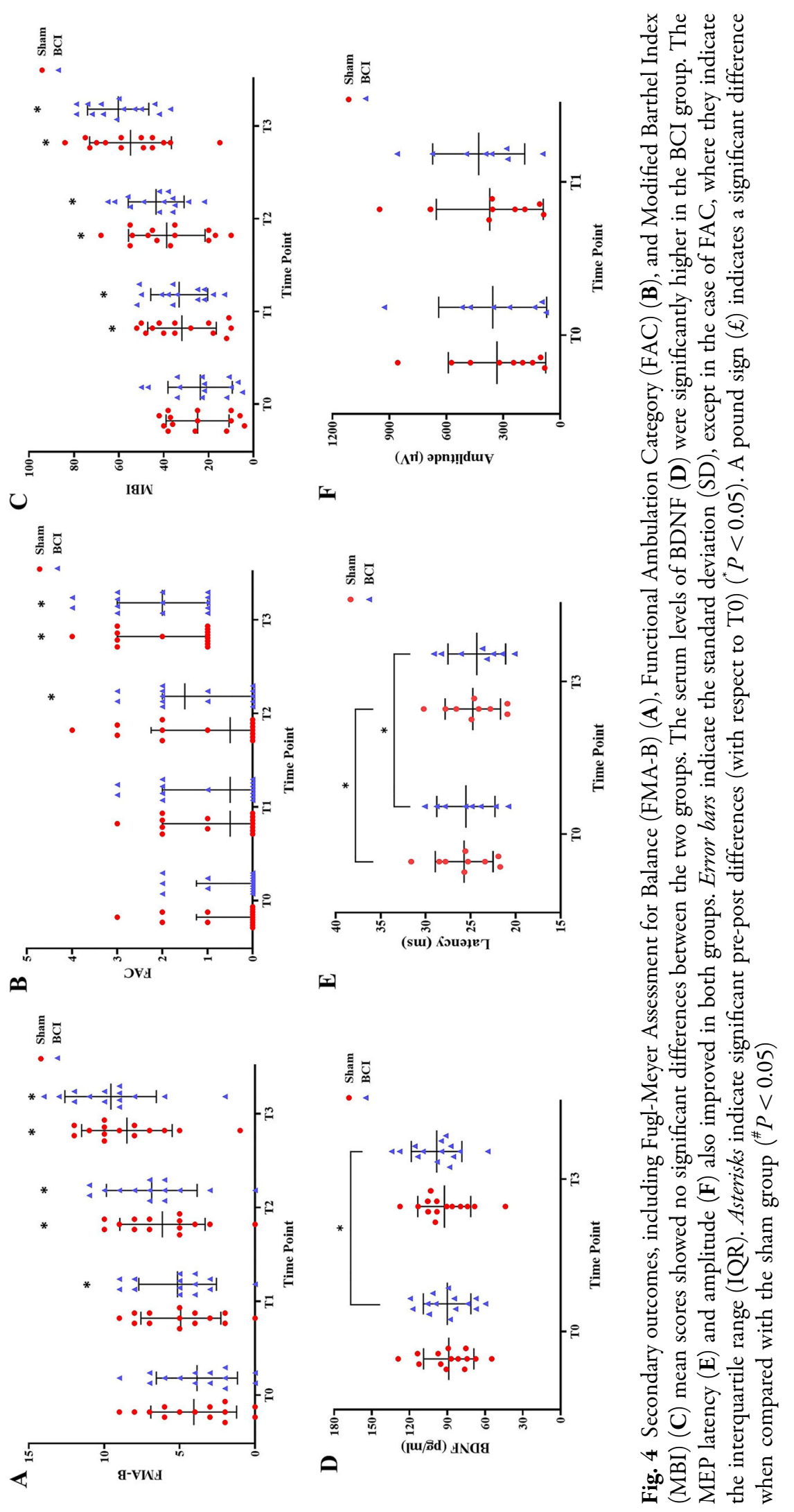


There were no significant differences between the two groups at any time point, although the effect size gradually increased (Cohen's $d \mathrm{~T} 1=-0.095, \mathrm{~T} 2=-0.161, \mathrm{~T} 3=-0.168)$. (Table 2; Fig. 3B).

\section{Secondary Outcomes}

Clinical scores (FMA-B, FAC, and MBI) were assessed at all four time points, Serum BDNF levels and neurophysiological parameters were measured or recorded before treatment (T0) and on the 4 th week (T3).

\section{FMA-B}

The interaction between time and intervention was not significant $\left(P=0.192, \eta^{2}=0.124\right)$. The FMA-B scores were significantly higher compared with baseline scores at T1 [MD -1.29 (95\% CI -2.54 to -0.04$)$, Cohen's $d=-0.764$, $P=0.041]$, T2 $[\mathrm{MD}-3.00(95 \% \mathrm{CI}-4.25$ to $-1.75), P<0.001$, Cohen's $d=-1.783]$, and T3 [MD -5.71 (95\% CI -6.96 to -4.46$)$, $P<0.001$, Cohen's $d=-3.396$ ] in the BCI group, while the scores in the sham group only increased significantly at T2 [MD -2.07 (95\% CI -3.05 to -1.09$)$, Cohen's $d=-1.574$, $P<0.001]$ and T3 [MD $-4.43(95 \% \mathrm{CI}-5.41$ to $-3.45)$, Cohen's $d=-3.365, P<0.001]$. There were no significant differences between the two groups at any of the three time points, although the effect size gradually increased (Cohen's $d \mathrm{~T} 1=-0.060, \mathrm{~T} 2=-0.174, \mathrm{~T} 3=-0.252)$. (Table 2; Fig. 4A).

\section{FAC}

The FAC scores were significantly higher compared with baseline scores at T2 $(P=0.008)$ and T3 $(P=0.001)$ in the BCI group. In contrast, in the sham group, they increased significantly only at T3 $(P<0.001)$. There were no significant differences between the two groups at any of the three time points $\left(P_{\mathrm{T} 1}=0.784, P_{\mathrm{T} 2}=0.771\right.$, $P_{\mathrm{T} 3}=0.363$ ). (Table 2; Fig. 4B).

\section{MBI}

The interaction between time and intervention was not significant $\left(P=0.401, \eta^{2}=0.065\right)$. Compared with the baseline MBI scores, the scores in both the sham and BCI groups increased significantly as the treatment progressed $\quad\left(P<0.001, \quad \eta^{2}=0.712 ; \quad P<0.001\right.$, $\left.\eta^{2}=0.843\right)$. The highest MBI scores were observed on the 4th week [MD - 29.86 (95\% CI 43.80 to -15.91$)$, Cohen's $d=-1.778$, $P<0.001 ;$ MD -36.50 (95\% CI -48.33 to - 24.67), Cohen's $d=-2.563, \quad P<0.001]$. However, there were no significant differences between the two groups at any time point $\left(P_{\mathrm{T} 1}=0.811, P_{\mathrm{T} 2}=0.441, P_{\mathrm{T} 3}=0.493\right.$, Cohen's $d \mathrm{~T} 1=-0.065, \mathrm{~T} 2=-0.212, \mathrm{~T} 3=-0.188)$ (Table 2; Fig. 4C).

\section{Serum BDNF Levels}

Serum BDNF levels were significantly higher in the BCI group after 4 weeks of treatment [MD -15.95 (95\% CI -23.84 to -8.06$)$, Cohen's $d=-1.167, P<0.001]$. In contrast, the difference was not significant in the sham group $(P=0.381$, Cohen's $d=0.242)$. At the end of the intervention, serum BDNF concentrations in the BCI group $(98.52 \pm 20.12 \mathrm{pg} / \mathrm{mL})$ were higher than in the sham group $(92.42 \pm 21.00 \mathrm{pg} / \mathrm{mL})$, although not significant $(P=0.092$, Cohen's $d=-0.661)$ (Table 2; Fig. 4D).

\section{Neurophysiological Parameters}

\section{MEP Latency}

Both the sham and BCI groups showed a significant reduction in latency after 4 weeks of treatment [MD $0.97 \quad(95 \%$ CI $0.69-1.24)$, Cohen's $d=2.707, P<0.001 ; \mathrm{MD}-1.21(95 \%$ CI 0.34-2.09), Cohen's $d=1.160, P=0.013$, respectively], with MEP latency reductions of $3.75 \%$ and $4.71 \%$, respectively. However, at the end of treatment, the latency values between the two groups did not show any significant difference $(P=0.776, \quad$ Cohen's $d=0.141)$ (Table 2; Fig. 4E).

\section{Maximal MEP Amplitude}

After training, the maximal MEP amplitude showed an increase in the BCI group [MD - 73.50 (95\% CI - 160.82 to 13.82), Cohen's $d=-0.704, P=0.087]$ and in the sham group 
$(P=0.143, \quad$ Cohen's $d=-0.541), \quad$ although neither were significantly different. Compared with the sham group, the maximal MEP amplitude increased by $15.59 \% \quad(P=0.659$, Cohen's $d=-0.219$ ) in the BCI group (Table 2; Fig. 4F).

\section{DISCUSSION}

Our study found that a 4-week training course with the BCI-controlled robot significantly increased LOCTA scores, and enhanced motor function of the lower extremities. These changes were accompanied by increased secretion of BDNF. This is the first report of an innovative approach based on SSMVEPs and training with a BCI-controlled robot which enables patients to exercise actively in the early phase after a stroke.

Although the rehabilitation of patients with stroke is a challenging task for doctors, interdisciplinary approaches offer novel solutions, and these include BCI-controlled devices $[26,27]$. BCI can be defined as a system which translates the brain activity patterns (typically measured by electroencephalography) into signals for interactive applications [28-30]. In this study, we propose a method named as active and passive co-stimulation, which could rebuild the loop-locked motor nerve pathway. Visual stimulation provides active stimulus to the nerve of motion control by mirror neuron, while the robot provides passive stimulus to the nerve of motion perception. By analyzing the EEG, the system could apperceive user's motor intention and use it to control the active and passive co-stimulation, which was a part of more complex BCI system involving motor imagery practice [31]. In our previous study, we utilized a special form of visual stimulation involving non-direction-specific motion reversals to elicit SSMVEPs for BCI applications $[15,32]$. This method can overcome problems of visual fatigue caused by uncomfortable light twinkling and contrast changes, as well as adaptation effects. We proposed an EEG-driven lower limb rehabilitation system for patients with stroke. By analyzing the EEG, the system can deduce the patient's motor intentionality and use that information to control the lower limb rehabilitation robot. Based on the previous study, the BCI-controlled robot training system was built, with visual stimulation, acquisition, EEG processing, and robot-assisted motion components [16]. We set different levels of training tasks for each patient, and, as the training level difficulty increased, the cognitive function requirements, such as memory and attention, also increased step by step.

After a stroke, motor dysfunction is always accompanied by cognitive impairment, which seriously affects the activities of daily living [21]. Many studies based on the principle of neurofeedback therapy [33] have reported that BCI-controlled systems can improve attention and other cognitive abilities [34]. For example, Lee et al. [35] developed games with a memorytraining component for the elderly population, and showed that they improved attention and memory. Kleih et al. [36] trained aphasic patients with a P300-BCI speller communication system, and showed that it improved aphasia recovery and neural plasticity by activating language circuits. In this study, we utilized a specially designed BCI-controlled robot training system for the rehabilitation of subacute stroke patients, and the initial results are encouraging. After 4 weeks of training, patients in the BCI group showed better cognitive function than the sham group, which is consistent with Edelman's report [37]. This result also indicates that the gradual improvement in cognitive function partly depends on the summative effects of training time, as reported by Carelli et al. [38]. The cognitive function assessment test used in this study was LOTCA, which measures a wide range of basic cognitive functions. Since our BCI training system required intensive attention, visuomotor organization, and memory abilities, training of these specific cognitive functions may be one of the reasons for the improvement in LOCTA scores [39].

Gait and balance impairments due to limb deficits after a stroke are one of the main determinants associated with poorer functional recovery $[40,41]$. Since the survival rates of stroke patients have increased, developing more effective treatments to improve walking is one 
of the major goals of stroke rehabilitation $[42,43]$. However, there is a lack of well-defined rehabilitation protocols. Intelligent, motor-driven devices for rehabilitation, or so-called rehabilitation robotics, represent an exciting new frontier with considerable potential to address these concerns. Several studies have shown that intensive training protocols with robotic devices combined with physical therapy produce comparable gains in motor function among acute or subacute stroke patients $[44,45]$. However, end-effector and exoskeletal devices only provide passive training, and patients cannot exercise spontaneously [46]. We developed a BCI-controlled robot for lower limb rehabilitation which allows subacute stroke patients to actively train their lower limbs. Our results demonstrated that FMA-LE scores in both the sham and BCI groups increased significantly with respect to baseline. However, there were no significant differences between the two groups at any time point, a result that is not completely in line with previous studies. A study of chronic stroke patients comparing robot treatment with traditional treadmill training in an intensive 4 -week protocol reported an advantage of the robot treatment, with significant improvements in gait speed, step length, and balance [47]. However, in our study, the results only appeared to be an enhancement in motor functions with no significant difference. Several reasons may account for this discrepancy, but the most important factors were the relatively short duration of the intervention and training time. We found that although there were no statistically significant differences between the two groups in the three time points, the effect size between them increased gradually, suggesting that significant differences would be seen with a longer training course. We plan to investigate this possibility in a future clinical trial.

BDNF is a neurotrophic factor with growthpromoting and neuronal plasticity effects on various neuronal populations after injury [9]. Several reports have demonstrated a relationship between BDNF levels and cognitive function in clinical trials [48]. For example, Miyamoto et al. [49] found that serum BDNF levels increased significantly after cognitive training, and Borror et al. [50] found that a change in BDNF levels in stroke patients after functional training was one of the key factors associated with improved cognitive function. Our results demonstrated that 4 weeks of training with a BCI-controlled robot significantly increased serum BDNF levels, which suggest that increasing levels of BDNF after training with a BCI-robot may explain the cognitive improvement in stroke patients.

MEP latency and amplitude are two important indicators of neuronal damage and of the relative integrity of the connecting pathways, especially of the corticospinal tract [51]. In the present study, we found significant pre-post differences in MEP latencies in both groups and a borderline significant difference in MEP amplitude in the BCI group with respect to baseline, and that these were positively correlated with cognitive and motor functions. However, MEP latency and amplitude were not significantly different between the two groups, and this was also consistent with the clinical evaluation. Although MEP latency and amplitude are two important parameters for evaluating neurophysiological changes [52], they do not always change in parallel. Latency is perhaps a more valuable parameter for evaluating the status of the connecting pathway, since MEP amplitude is more easily influenced by age, the resting state of the muscle, etc. Interestingly, although MEPs were not initially detected in five patients from the sham group and in six from the BCI group, we finally succeeded in detecting them in three patients, suggesting that functional recovery may occur earlier than neurophysiological changes.

Our present study has several potential limitations. First, the number of participants was relatively small, which may increase the risk of type-II errors. Second, limited by objective conditions, the duration of the intervention was relatively short, and this may influence the motor function recovery results. The fact that the effect size between the two groups increased with the duration of the intervention suggests that we may see significant differences with more prolonged interventions. Finally, other tests, such as functional magnetic resonance imaging and functional near-infrared imaging, 
are needed to understand the mechanisms underlying the beneficial effects of BCI training.

\section{CONCLUSIONS}

This is the first study of lower limb rehabilitation in patients with subacute stroke using a BCI-controlled robotic device and a Newton's ring to elicit SSMVEPs. We provide evidence that training with a BCI-controlled robot, combined with traditional PT, is an effective strategy to promote cognitive recovery and that it enhances motor function of the lower extremities in stroke patients. These changes were accompanied by increased secretion of BDNF.

\section{ACKNOWLEDGEMENTS}

We thank Yuan Shen for the helping in statistical analysis and we also thank all the study participants and their families.

Funding. The study and the Rapid Service Fee were supported by grants from the National Natural Science Foundation (81100932, $82072534,91420301)$, the Shaanxi Science and Technology Foundation (2021JM-232, 2020KW050, 2018ZDCXL-GY-06-01).

Authorship. All named authors meet the ICMJE criteria for authorship for this article, take responsibility for the integrity of the work as a whole, and have given their approval for this version to be published.

Authors' Contributions. Professor Yuan had full access to all of the data in the study and takes responsibility for the integrity of the data and the accuracy of the data analysis. Concept and design: Yuan, Zhao, Xu, Zhang, Mou. Acquisition, analysis, or interpretation of data: Yuan, Zhao, Ju, Sun, Xi, Wang, Sun, Mou. Drafting of the manuscript: Yuan, Zhao, Ju, Jiang, Xi, Li, Zhang. Statistical analysis: Yuan, Zhao, Jiang. Administrative, technical, or material support: Li, Xie, Zhang, Xu, Zhang.
Disclosures. Chen-Guang Zhao, Fen Ju, Wei Sun, Shan Jiang, Xiao Xi, Hong Wang, XiaoLong Sun, Min Li, Jun Xie, Kai Zhang, GuangHua Xu, Si-Cong Zhang, Xiang Mou, Hua Yuan report no competing interests.

Compliance with Ethics Guidelines. The study was approved by the Institute Ethics Committee of Xijing Hospital (KY20172051), adhered to the guidelines of the Declaration of Helsinki, and was registered in the Chinese Clinical Trial Registry (ChiCTR-INR-17012874). Informed consent was received to participate and also include the statement we provided concerning written informed consent for the publication and for the photograph (including Fig. 1A) included in the paper.

Data Availability. The data that support the findings of this study are available from the corresponding author upon reasonable request.

Open Access. This article is licensed under a Creative Commons Attribution-NonCommercial 4.0 International License, which permits any non-commercial use, sharing, adaptation, distribution and reproduction in any medium or format, as long as you give appropriate credit to the original author(s) and the source, provide a link to the Creative Commons licence, and indicate if changes were made. The images or other third party material in this article are included in the article's Creative Commons licence, unless indicated otherwise in a credit line to the material. If material is not included in the article's Creative Commons licence and your intended use is not permitted by statutory regulation or exceeds the permitted use, you will need to obtain permission directly from the copyright holder. To view a copy of this licence, visit http://creativecommons.org/licenses/by$\mathrm{nc} / 4.0 /$.

\section{REFERENCES}

1. White JH, Alston MK, Marquez JL, Sweetapple AL, Pollack MR, Attia J, et al. Community-dwelling stroke survivors: function is not the whole story with quality of life. Arch Phys Med Rehabil. 
2007;88(9):1140-6. https://doi.org/10.1016/j.apmr. 2007.06.003.

2. Zhou M, Wang H, Zhu J, Chen W, Wang L, Liu S, et al. Cause-specific mortality for 240 causes in china during 1990-2013: a systematic subnational analysis for the global burden of disease study 2013. Lancet. 2016;387(10015):251-72. https://doi.org/ 10.1016/S0140-6736(15)00551-6.

3. GBD 2016 Causes of Death Collaborators. Global, regional, and national age-sex specific mortality for 264 causes of death, 1980-2016: a systematic analysis for the global burden of disease study 2016 . Lancet. 2017;390(10100):1151-210. https://doi. org/10.1016/S0140-6736(17)32152-9.

4. Stinear CM, Lang CE, Zeiler S, Byblow WD. Advances and challenges in stroke rehabilitation. Lancet Neurol. 2020;19(4):348-60. https://doi.org/ 10.1016/S1474-4422(19)30415-6.

5. Pollock A, George BS, Fenton M, Firkins L. Top 10 research priorities relating to life after stroke-consensus from stroke survivors, caregivers, and health professionals. Int J Stroke. 2014;9(3):313-20. https://doi.org/10.1111/j.1747-4949.2012.00942.x.

6. Smithard DG. Management of stroke: acute, rehabilitation and long-term care. Hosp Med. 2003;64(11):666-72. https://doi.org/10.12968/ hosp.2003.64.11.2349.

7. Winstein CJ, Stein J, Arena R, Bates B, Cherney LR, Cramer SC, et al. Guidelines for adult stroke rehabilitation and recovery: a guideline for healthcare professionals from the American Heart Association/ American Stroke Association. Stroke. 2016;47(6): e98-169. 0000000000000098 .

8. Starostka-Tatar A, Łabuz-Roszak B, Skrzypek M, Gąsior M, Gierlotka M. Definition and treatment of stroke over the centuries. Wiad Lek. 2017;70(5): 982-7.

9. de Sousa Fernandes MS, Ordônio TF, Santos GCJ, Santos LER, Calazans CT, Gomes DA, et al. Effects of physical exercise on neuroplasticity and brain function: a systematic review in human and animal studies. Neural Plast. 2020;2020:8856621. https:// doi.org/10.1155/2020/8856621.

10. Bockbrader MA, Francisco G, Lee R, Olson J, Solinsky $\mathrm{R}$, Boninger ML. Brain computer interfaces in rehabilitation medicine. PMR. 2018;10(9 Suppl 2): S233-43. https://doi.org/10.1016/j.pmrj.2018.05. 028.

11. Chaudhary U, Birbaumer N, Ramos-Murguialday A. Brain-computer interfaces for communication and rehabilitation. Nat Rev Neurol. 2016;12(9):513-25. https://doi.org/10.1038/nrneurol.2016.113.

12. Yan W, Xu G, Li M, Xie J, Han C, Zhang S, et al. Steady-state motion visual evoked potential (SSMVEP) based on equal luminance colored enhancement. PLoS ONE. 2017;12(1): e0169642. https://doi.org/10.1371/journal.pone.0169642.

13. Yan W, Xu G, Chen L, Zheng X. Steady-state motion visual evoked potential (SSMVEP) enhancement method based on time-frequency image fusion. Comput Intell Neurosci. 2019;2019: 9439407. https://doi.org/10.1155/2019/9439407.

14. Han C, Xu G, Xie J, Li M, Zhang S, Luo A. An eightytarget high-speed Chinese BCI speller. Annu Int Conf IEEE Eng Med Biol Soc. 2017;2017:1652-5. https://doi.org/10.1109/EMBC.2017.8037157.

15. Xie J, Xu G, Wang J, Zhang F, Zhang Y. Steady-state motion visual evoked potentials produced by oscillating newton's rings: implications for braincomputer interfaces. PLOS ONE. 2012;7(6): e39707. https://doi.org/10.1371/journal.pone.0039707.

16. Zhang X, Xu G, Xie J, Li M, Pei W, Zhang J. An EEGdriven lower limb rehabilitation training system for active and passive co-stimulation. Annu Int Conf IEEE Eng Med Biol Soc. 2015;2015:4582-5. https:// doi.org/10.1109/EMBC.2015.7319414.

17. Zhao CG, Sun W, Ju F, Wang H, Sun XL, Mou X, et al. Analgesic effects of directed repetitive transcranial magnetic stimulation in acute neuropathic pain after spinal cord injury. Pain Med. 2020;21(6): 1216-23. https://doi.org/10.1093/pm/pnz290.

18. Shirota Y, Ohminami S, Tsutsumi R, Terao Y, Ugawa $\mathrm{Y}$, Tsuji S, et al. Increased facilitation of the primary motor cortex in de novo parkinson's disease. Parkinsonism Relat Disord. 2019;66:125-9. https:// doi.org/10.1016/j.parkreldis.2019.07.022.

19. Inukai Y, Saito K, Sasaki R, Tsuiki S, Miyaguchi S, Kojima $S$, et al. Comparison of three non-invasive transcranial electrical stimulation methods for increasing cortical excitability. Front Hum Neurosci. 2016;10:668. https://doi.org/10.3389/fnhum. 2016.00668 .

20. Rojo-Mota G, Pedrero-Pérez EJ, Ruiz-Sánchez de León JM, León-Frade I, Aldea-Poyo P, AlonsoRodríguez $\mathrm{M}$, et al. Loewenstein occupational therapy cognitive assessment to evaluate people with addictions. Occup Ther Int. 2017;2017: 2750328. https://doi.org/10.1155/2017/2750328.

21. Schwartz Y, Averbuch S, Katz N, Sagiv A. Validity of the Functional Loewenstein Occupational Therapy Cognitive Assessment (FLOTCA). Am J Occup Ther. 
2016;70(1):7001290010p1-7. https://doi.org/10. 5014/ajot.2016.016451.

22. Almomani F, Avi-Itzhak T, Demeter N, Josman N, Al-Momani MO. Construct validity and internal consistency reliability of the Loewenstein occupational therapy cognitive assessment (LOTCA). BMC Psychiatry. 2018;18(1):184. https://doi.org/10. 1186/s12888-018-1776-X.

23. Mustafaoglu R, Erhan B, Yeldan I, Gunduz B, Tarakci E. Does robot-assisted gait training improve mobility, activities of daily living and quality of life in stroke? A single-blinded, randomized controlled trial. Acta Neurol Belg. 2020;120(2):335-44. https:// doi.org/10.1007/s13760-020-01276-8.

24. Chang WH, Kim MS, Huh JP, Lee PK, Kim YH. Effects of robot-assisted gait training on cardiopulmonary fitness in subacute stroke patients: a randomized controlled study. Neurorehabil Neural Repair. 2012;26(4):318-24. https://doi.org/10.1177/ 1545968311408916.

25. Kim J, Kim DY, Chun MH, Kim SW, Jeon HR, Hwang $\mathrm{CH}$, et al. Effects of robot-(morning walk $\left.{ }^{\circledR}\right)$ assisted gait training for patients after stroke: a randomized controlled trial. Clin Rehabil. 2019;33(3):516-23. https://doi.org/10.1177/ 0269215518806563.

26. Grefkes C, Fink GR. Recovery from stroke: current concepts and future perspectives. Neurol Res Pract. 2020;2:17. https://doi.org/10.1186/s42466-02000060-6.

27. Aprile I, Guardati G, Cipollini V, Papadopoulou D, Mastrorosa A, Castelli L, et al. Robotic rehabilitation: an opportunity to improve cognitive functions in subjects with stroke. An explorative study. Front Neurol. 2020;11: 588285. https://doi.org/10. 3389/fneur.2020.588285.

28. Lotte F, Bougrain L, Cichocki A, Clerc M, Congedo M, Rakotomamonjy A, et al. A review of classification algorithms for EEG-based brain-computer interfaces: a 10 year update. J Neural Eng. 2018;15(3): 031005. https://doi.org/10.1088/17412552/aab2f2.

29. Roc A, Pillette L, Mladenovic J, Benaroch C, $\mathrm{N}^{\prime}$ Kaoua B, Jeunet C, et al. A review of user training methods in brain computer interfaces based on mental tasks. J Neural Eng. 2020. https://doi.org/10. 1088/1741-2552/abca17 (Online ahead of print).

30. Wolpaw JR, Millán JDR, Ramsey NF. Brain-computer interfaces: definitions and principles. Handb Clin Neurol. 2020;168:15-23. https://doi.org/10. 1016/B978-0-444-63934-9.00002-0.
31. Pichiorri F, Mattia D. Brain-computer interfaces in neurologic rehabilitation practice. Handb Clin Neurol. 2020;168:101-16. https://doi.org/10.1016/ B978-0-444-63934-9.00009-3.

32. Zhang X, Xu G, Mou X, Ravi A, Li M, Wang Y, et al. A convolutional neural network for the detection of asynchronous steady state motion visual evoked potential. IEEE Trans Neural Syst Rehabil Eng. 2019;27(6):1303-11. https://doi.org/10.1109/ TNSRE.2019.2914904.

33. Yoo IG. Electroencephalogram-based neurofeedback training in persons with stroke: a scoping review in occupational therapy. Neuro Rehabilit. 2021;48(1):9-18. https://doi.org/10.3233/NRE201579.

34. Belkacem AN, Jamil N, Palmer JA, Ouhbi S, Chen C. Brain computer interfaces for improving the quality of life of older adults and elderly patients. Front Neurosci. 2020;14:692. https://doi.org/10.3389/ fnins.2020.00692.

35. Lee TS, Goh SJ, Quek SY, Phillips R, Guan C, Cheung YB, et al. A brain-computer interface based cognitive training system for healthy elderly: a randomized control pilot study for usability and preliminary efficacy. PLoS ONE. 2013;8(11): e79419. https://doi.org/10.1371/journal.pone. 0079419

36. Kleih SC, Gottschalt L, Teichlein E, Weilbach FX. Toward a p300 based brain-computer interface for aphasia rehabilitation after stroke: presentation of theoretical considerations and a pilot feasibility study. Front Hum Neurosci. 2016;10:547. https:// doi.org/10.3389/fnhum.2016.00547.

37. Edelman BJ, Meng J, Gulachek N, Cline CC, He B. Exploring cognitive flexibility with a noninvasive bci using simultaneous steady-state visual evoked potentials and sensorimotor rhythms. IEEE Trans Neural Syst Rehabil Eng. 2018;26(5):936-47. https://doi.org/10.1109/TNSRE.2018.2817924.

38. Carelli L, Solca F, Faini A, Meriggi P, Sangalli D, Cipresso P, et al. Brain-computer interface for clinical purposes: cognitive assessment and rehabilitation. Biomed Res Int. 2017;2017:1695290. https:// doi.org/10.1155/2017/1695290.

39. Sprague SA, McBee MT, Sellers EW. The effects of working memory on brain-computer interface performance. Clin Neurophysiol. 2016;127(2): 1331-41. https://doi.org/10.1016/j.clinph.2015.10. 038.

40. Pérez-de la Cruz S. Comparison between three therapeutic options for the treatment of balance and gait in stroke: a randomized controlled trial. Int 
J Environ Res Public Health. 2021;18(2):426. https://doi.org/10.3390/ijerph18020426.

41. Wade DT. Impact commentaries. Functional abilities after stroke: measurement, natural history and prognosis. J Neurol Neurosurg Psychiatry. 2012;83(8):770. https://doi.org/10.1136/jnnp2011-301689.

42. Koch G, Bonnì S, Casula EP, Iosa M, Paolucci S, Pellicciari MC, et al. Effect of cerebellar stimulation on gait and balance recovery in patients with hemiparetic stroke: a randomized clinical trial. JAMA Neurol. 2019;76(2):170-8. https://doi.org/10. 1001/jamaneurol.2018.3639.

43. Pillette L, Lotte F, N'Kaoua B, Joseph PA, Jeunet C, Glize B. Why we should systematically assess, control and report somatosensory impairments in BCIbased motor rehabilitation after stroke studies. Neuroimage Clin. 2020;28: 102417. https://doi.org/ 10.1016/j.nicl.2020.102417.

44. Moucheboeuf G, Griffier R, Gasq D, Glize B, Bouyer L, Dehail P, et al. Effects of robotic gait training after stroke: a meta-analysis. Ann Phys Rehabil Med. 2020;63(6):518-34. https://doi.org/10.1016/j. rehab.2020.02.008.

45. Kakuda W. Future directions of stroke rehabilitation. Rinsho Shinkeigaku. 2020;60(3):181-6. https://doi.org/10.5692/clinicalneurol.cn-001399.

46. Louie DR, Eng JJ. Powered robotic exoskeletons in post-stroke rehabilitation of gait: a scoping review. J Neuroeng Rehabil. 2016;13(1):53. https://doi.org/ 10.1186/s12984-016-0162-5.
47. Bang DH, Shin WS. Effects of robot-assisted gait training on spatiotemporal gait parameters and balance in patients with chronic stroke: a randomized controlled pilot trial. NeuroRehabilitation. 2016;38(4):343-9. https://doi.org/10.3233/NRE161325 .

48. Titus J, Bray NW, Kamkar N, Camicioli R, Nagamatsu LS, Speechley M, et al. The role of physical exercise in modulating peripheral inflammatory and neurotrophic biomarkers in older adults: a systematic review and meta-analysis. Mech Ageing Dev. 2021;194: 111431. https://doi.org/10.1016/j. mad.2021.111431.

49. Miyamoto T, Hashimoto S, Yanamoto H, Ikawa M, Nakano Y, Sekiyama T, et al. Response of brainderived neurotrophic factor to combining cognitive and physical exercise. Eur J Sport Sci. 2018;18(8): 1119-27. https://doi.org/10.1080/17461391.2018. 1470676 .

50. Borror A. Brain-derived neurotrophic factor mediates cognitive improvements following acute exercise. Med Hypotheses. 2017;106:1-5. https://doi. org/10.1016/j.mehy.2017.06.024.

51. Nguyen DTA, Rissanen SM, Julkunen P, Kallioniemi E, Karjalainen PA. Principal component regression on motor evoked potential in single-pulse transcranial magnetic stimulation. IEEE Trans Neural Syst Rehabil Eng. 2019;27(8):1521-8. https://doi. org/10.1109/TNSRE.2019.2923724.

52. Hanajima R, Ugawa Y. Triad TMS of the human motor cortex. Neurosci Res. 2020;156:245-9. https://doi.org/10.1016/j.neures.2019.11.005. 